International Journal of Pure and Applied Mathematics

Volume 85 No. 3 2013, 609-627

ISSN: 1311-8080 (printed version); ISSN: 1314-3395 (on-line version)

url: http://www.ijpam.eu

doi: http://dx.doi.org/10.12732/ijpam.v85i3.15

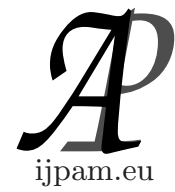

\title{
UPPER SEMICONTINUOUS \\ QUANTUM STOCHASTIC DIFFERENTIAL INCLUSIONS
}

M.O. Ogundiran

Department of Mathematics

Obafemi Awolowo University

Ile-Ife, NIGERIA

\begin{abstract}
This paper is concerned with the existence of solutions of quantum stochastic differential inclusions (QSDI) whose coefficients are upper semicontinuous. Our results are achieved within the framework of the HudsonParthasarathy formulation of quantum stochastic calculus.
\end{abstract}

AMS Subject Classification: $81 \mathrm{~S} 25$

Key Words: upper semicontinuous multivalued maps, partition of unity, quantum stochastic processes

\section{Introduction}

The problem of existence of solution of quantum stochastic differential inclusions in which the coefficients involved are upper semicontinuous multivalued stochastic processes is the aim of this paper. In [7] the existence of solutions of quantum stochastic differential inclusions with Lipschitzian coefficients lying in certain locally convex spaces was established. The problem of quantum stochastic control with discontinuous coefficients can be reformulated as quantum stochastic differential inclusions. The quantum stochastic differential inclusions formed in this case may have discontinuous coefficients which may be upper semicontinuous. As described in [1], in some cases, the upper semicontinuous multivalued maps so formed may be convex and compact. But this work is concerned with the case of coefficients which are upper semicontinuous multivalued sesquilinear forms having minimal selections lying in some compact

Received: $\quad$ March 21, 2013

(c) 2013 Academic Publications, Ltd. url: www.acadpubl.eu 
subsets of complex numbers. For a classical differential inclusions an existence of solutions of this form was established in [1]. We establish a non commutative generalization of this result. It is noteworthy that upper semicontinuous QSDI have found applications in quantum stochastic control [14], in quantum dynamical systems and other areas in quantum field theory.

The rest of the paper is organized as follows: Section 2 is devoted to the preliminaries necessary for the understanding of the work. This includes a comprehensive description of the QSDI and associated upper semicontinuous multivalued maps. Section 3 deals with the main existence results. An approximate selection result was established in Subsection 3.3. The selection result facilitated the establishment of the main results in Section 3.4.

\section{Preliminaries}

In this section we assemble together the definitions and notations which shall be employed in what follows. Adopting the notation and formulations in [7], we define upper semicontinuous maps and the associated multivalued sesquilinear forms.

\subsection{Notations}

In what follows, if $U$ is a topological space, we denote by $\operatorname{clos}(U)$, the collection of all non-empty closed subsets of $U$.

To each pair $(D, H)$ consisting of a pre-Hilbert space $D$ and its completion $H$, we associate the set $L_{w}^{+}(D, H)$ of all linear maps $x$ from $D$ into $H$, with the property that the domain of the operator adjoint contains $D$. The members of $L_{w}^{+}(D, H)$ are densely-defined linear operators on $H$ which do not necessarily leave $D$ invariant and $L_{w}^{+}(D, H)$ is a linear space when equipped with the usual notions of addition and scalar multiplication.

To $H$ corresponds a Hilbert space $\Gamma(H)$ called the boson Fock space determined by $H$. A natural dense subset of $\Gamma(H)$ consists of linear space generated by the set of exponential vectors(Guichardet, [12]) in $\Gamma(H)$ of the form

$$
e(f)=\bigoplus_{n=0}^{\infty}(n !)^{-\frac{1}{2}} \bigotimes^{n} f, \quad f \in H
$$

where $\otimes^{0} f=1$ and $\bigotimes^{n} f$ is the $n$-fold tensor product of $f$ with itself for $n \geq 1$. 
In what follows, $\mathbb{D}$ is some pre-Hilbert space whose completion is $\mathcal{R}$ and $\gamma$ is a fixed Hilbert.

$L_{\gamma}^{2}\left(\mathbb{R}_{+}\right)\left(\right.$resp. $L_{\gamma}^{2}([0, t))$, resp. $\left.L_{\gamma}^{2}([t, \infty)) t \in \mathbb{R}_{+}\right)$is the space of square integrable $\gamma$-valued maps on $\mathbb{R}_{+}$(resp. $[0, t)$, resp. $\left.[t, \infty)\right)$.

The inner product of the Hilbert space $\mathcal{R} \otimes \Gamma\left(L_{\gamma}^{2}\left(\mathbb{R}_{+}\right)\right)$will be denoted by $\langle.,$.$\rangle and \|$.$\| the norm induced by \langle.,$.$\rangle .$

Let $\mathbb{E}, \mathbb{E}_{t}$ and $\mathbb{E}^{t}, t>0$ be linear spaces generated by the exponential vectors in Fock spaces $\Gamma\left(L_{\gamma}^{2}\left(\mathbb{R}_{+}\right)\right), \Gamma\left(L_{\gamma}^{2}([0, t))\right)$ and $\Gamma\left(L_{\gamma}^{2}([t, \infty))\right)$ respectively ;

$$
\begin{aligned}
\mathcal{A} & \equiv L_{w}^{+}\left(\mathbb{D} \underline{\otimes} \mathbb{E}, \mathcal{R} \otimes \Gamma\left(L_{\gamma}^{2}\left(\mathbb{R}_{+}\right)\right)\right) \\
\mathcal{A}_{t} & \equiv L_{w}^{+}\left(\mathbb{D} \underline{\otimes} \mathbb{E}_{t}, \mathcal{R} \otimes \Gamma\left(L_{\gamma}^{2}([0, t))\right)\right) \otimes \mathbb{I}^{t} \\
\mathcal{A}^{t} & \equiv \mathbb{I}_{t} \otimes L_{w}^{+}\left(\mathbb{E}^{t}, \Gamma\left(L_{\gamma}^{2}([t, \infty))\right)\right), t>0
\end{aligned}
$$

where $\underline{\otimes}$ denotes algebraic tensor product and $\mathbb{I}_{t}\left(\right.$ resp. $\left.\mathbb{I}^{t}\right)$ denotes the identity map on $\left.\mathcal{R} \otimes \Gamma\left(L_{\gamma}^{2}([0, t))\right)\right)\left(\operatorname{resp} . \Gamma\left(L_{\gamma}^{2}([t, \infty))\right)\right), t>0$

For every $\eta, \xi \in \mathbb{D} \underline{\otimes} \mathbb{E}$ define

$$
\|x\|_{\eta \xi}=|\langle\eta, x \xi\rangle|, \quad x \in \mathcal{A}
$$

then the family of seminorms

$$
\left\{\|\cdot\|_{\eta \xi}: \eta, \xi \in \mathbb{D} \underline{\otimes} \mathbb{E}\right\}
$$

generates a topology $\tau_{w}$, weak topology.

The completion of the locally convex $\operatorname{spaces}\left(\mathcal{A}, \tau_{w}\right),\left(\mathcal{A}_{t}, \tau_{w}\right)$ and $\left(\mathcal{A}^{t}, \tau_{w}\right)$ are respectively denoted by $\widetilde{\mathcal{A}}, \widetilde{\mathcal{A}}_{t}$ and $\widetilde{\mathcal{A}}^{t}$.

We define the Hausdorff topology on $\operatorname{clos}(\widetilde{\mathcal{A}})$ as follows:

For $x \in \widetilde{\mathcal{A}}, \mathcal{M}, \mathcal{N} \in \operatorname{clos}(\widetilde{\mathcal{A}})$ and $\eta, \xi \in \mathbb{D} \underline{\otimes} \mathbb{E}$, define

$$
\rho_{\eta \xi}(\mathcal{M}, \mathcal{N}) \equiv \max \left(\delta_{\eta \xi}(\mathcal{M}, \mathcal{N}), \delta_{\eta \xi}(\mathcal{N}, \mathcal{M})\right)
$$

where

$$
\begin{array}{r}
\delta_{\eta \xi}(\mathcal{M}, \mathcal{N}) \equiv \sup _{x \in \mathcal{M}} \mathbf{d}_{\eta \xi}(x, \mathcal{N}) \text { and } \\
\mathbf{d}_{\eta \xi}(x, \mathcal{N}) \equiv \inf _{y \in \mathcal{N}}\|x-y\|_{\eta \xi}
\end{array}
$$

The Hausdorff topology which shall be employed in what follows, denoted by, $\tau_{H}$, is generated by the family of pseudometrics $\left\{\rho_{\eta \xi}():. \eta, \xi \in \mathbb{D} \underline{\otimes} \mathbb{E}\right\}$

Moreover, if $\mathcal{M} \in \operatorname{clos}(\widetilde{\mathcal{A}})$, then $\|\mathcal{M}\|_{\eta \xi}$ is defined by

$$
\|\mathcal{M}\|_{\eta \xi} \equiv \rho_{\eta \xi}(\mathcal{M},\{0\})
$$


for arbitrary $\eta, \xi \in \mathbb{D} \underline{\otimes} \mathbb{E}$.

For $A, B \in \operatorname{clos}(\mathbb{C})$ and $x \in \mathbb{C}$, a complex number, define

$$
\begin{aligned}
\mathbf{d}(x, B) & \equiv \inf _{y \in B}|x-y| \\
\delta(A, B) & \equiv \sup _{x \in A} \mathbf{d}(x, B) \\
\text { and } \rho(A, B) & \equiv \max (\delta(A, B), \delta(B, A)) .
\end{aligned}
$$

Then $\rho$ is a metric on $\operatorname{clos}(\mathbb{C})$ and induces a metric topology on the space.

Let $I \subseteq \mathbb{R}_{+}$. A stochastic process indexed by $I$ is an $\widetilde{\mathcal{A}}$-valued measurable map on $I$.

A stochastic process $X$ is called adapted if $X(t) \in \widetilde{\mathcal{A}}_{t}$ for each $t \in I$.

We write $\operatorname{Ad}(\widetilde{\mathcal{A}})$ for the set of all adapted stochastic processes indexed by $I$.

Definition 2.1. A member $X$ of $\operatorname{Ad}(\widetilde{\mathcal{A}})$ is called

(i) weakly absolutely continuous if the map $t \mapsto\langle\eta, X(t) \xi\rangle, t \in I$ is absolutely continuous for arbitrary $\eta, \xi \in \mathbb{D} \underline{\otimes} \mathbb{E}$

(ii) locally absolutely p-integrable if $\|X(.)\|_{\eta \xi}^{p}$ is Lebesgue -measurable and integrable on $[0, t) \subseteq I$ for each $t \in I$ and arbitrary $\eta, \xi \in \mathbb{D} \otimes \mathbb{E}$.

We denote by $\operatorname{Ad}(\widetilde{\mathcal{A}})_{w a c}\left(\operatorname{resp} . L_{l o c}^{p}(\widetilde{\mathcal{A}})\right)$ the set of all weakly, absolutely continuous(resp. locally absolutely p-integrable) members of $\operatorname{Ad}(\widetilde{\mathcal{A}})$.

Stochastic integrators: Let $L_{\gamma, \text { loc }}^{\infty}\left(\mathbb{R}_{+}\right)\left[\operatorname{resp} . L_{B(\gamma), \text { loc }}^{\infty}\left(\mathbb{R}_{+}\right)\right]$be the linear space of all measurable, locally bounded functions from $\mathbb{R}_{+}$to $\gamma[$ resp. to $\mathrm{B}(\gamma)$, the Banach space of bounded endomorphisms of $\gamma]$. If $f \in L_{\gamma, l o c}^{\infty}\left(\mathbb{R}_{+}\right)$and $\pi \in$ $L_{B(\gamma), \text { loc }}^{\infty}\left(\mathbb{R}_{+}\right)$, then $\pi f$ is the member of $L_{\gamma, \text { loc }}^{\infty}\left(\mathbb{R}_{+}\right)$given by $(\pi f)(t)=\pi(t) f(t)$, $t \in \mathbb{R}_{+}$.

For $f \in L_{\gamma}^{2}(\mathbb{R})_{+}$and $\pi \in L_{B(\gamma), \text { loc }}^{\infty}\left(\mathbb{R}_{+}\right)$; the annihilation, creation and gauge operators, $a(f), a^{+}(f)$ and $\lambda(\pi)$ in $L_{w}^{+}\left(\mathbb{D}, \Gamma\left(L_{\gamma}^{2}(\mathbb{R})_{+}\right)\right)$respectively, are defined as:

$$
\begin{aligned}
a(f) \mathbf{e}(g) & =\langle f, g\rangle_{L_{\gamma}^{2}\left(\mathbb{R}_{+}\right)} \mathbf{e}(g) \\
a^{+}(f) \mathbf{e}(g) & =\left.\frac{d}{d \sigma} \mathbf{e}(g+\sigma f)\right|_{\sigma=0} \\
\lambda(\pi) \mathbf{e}(g) & =\left.\frac{d}{d \sigma} \mathbf{e}\left(e^{\sigma \pi} f\right)\right|_{\sigma=0}
\end{aligned}
$$

$g \in L_{\gamma}^{2}(\mathbb{R})_{+}$ 
For arbitrary $f \in L_{\gamma, l o c}^{\infty}\left(\mathbb{R}_{+}\right)$and $\pi \in L_{B(\gamma), \text { loc }}^{\infty}\left(\mathbb{R}_{+}\right)$, they give rise to the operator-valued maps $A_{f}, A_{f}^{+}$and $\Lambda_{\pi}$ defined by:

$$
\begin{aligned}
A_{f}(t) & \equiv a\left(f \chi_{[0, t)}\right) \\
A_{f}^{+}(t) & \equiv a^{+}\left(f \chi_{[0, t)}\right) \\
\Lambda_{\pi}(t) & \equiv \lambda\left(\pi \chi_{[0, t)}\right)
\end{aligned}
$$

$t \in \mathbb{R}_{+}$, where $\chi_{I}$ denotes the indicator function of the Borel set $I \subseteq \mathbb{R}_{+}$. The maps $A_{f}, A_{f}^{+}$and $\Lambda_{\pi}$ are stochastic processes, called annihilation, creation and gauge processes, respectively, when their values are identified with their ampliations on $\mathcal{R} \otimes \Gamma\left(L_{\gamma}^{2}\left(\mathbb{R}_{+}\right)\right)$. These are the stochastic integrators in Hudson and Parthasarathy[15] formulation of boson quantum stochastic integration.

For processes $p, q, u, v \in L_{l o c}^{2}(\widetilde{\mathcal{A}})$, the quantum stochastic integral:

$$
\int_{t_{0}}^{t}\left(p(s) d \Lambda_{\pi}(s)+q(s) d A_{f}(s)+u(s) d A_{g}^{+}(s)+v(s) d s\right), \quad t_{0}, t \in \mathbb{R}_{+}
$$

is interpreted in the sense of Hudson-Parthasarathy[15]

\subsection{Quantum Stochastic Differential Inclusions}

Definition 2.2. (a) By a multivalued stochastic process indexed by $I \subseteq$ $\mathbb{R}_{+}$we mean a multifunction on $I$ with values in $\operatorname{clos}(\widetilde{\mathcal{A}})$.

(b) If $\Phi$ is a multivalued stochastic process indexed by $I \subseteq \mathbb{R}_{+}$, then a selection of $\Phi$ is a stochastic process $X: I \rightarrow \widetilde{\mathcal{A}}$ with the property that $X(t) \in$ $\Phi(t)$ for almost all $t \in I$.

(c) A multivalued stochastic process $\Phi$ will be called (i) adapted if $\Phi(t) \subseteq \widetilde{\mathcal{A}}_{t}$ for each $t \in \mathbb{R}_{+}$; (ii) measurable if $t \mapsto \mathbf{d}_{\eta \xi}(x, \Phi(t))$ is measurable for arbitrary $x \in \widetilde{\mathcal{A}}, \eta, \xi \in(\mathbb{D} \underline{\otimes} \mathbb{E})$

(iii) locally absolutely p-integrable if $t \mapsto\|\Phi(t)\|_{\eta \xi}, t \in \mathbb{R}_{+}$lie in $L_{l o c}^{p}(I)$ for arbitrary $\eta, \xi \in(\mathbb{D} \underline{\otimes} \mathbb{E})$

For $p \in(0, \infty)$ and $I \subseteq \mathbb{R}_{+}$, the set of all locally absolutely p-integrable multivalued stochastic processes will be denoted by $L_{l o c}^{p}(\widetilde{\mathcal{A}})_{m v s}$. Denote by $L_{l o c}^{p}(I \times \widetilde{\mathcal{A}})_{m v s}$ the set of maps $\Phi: I \times \widetilde{\mathcal{A}} \rightarrow \operatorname{clos}(\widetilde{\mathcal{A}})$ such that $t \mapsto \Phi(t, X(t))$, $t \in I$, lies in $L_{l o c}^{p}(\widetilde{\mathcal{A}})_{m v s}$ for every $X \in L_{l o c}^{p}(\widetilde{\mathcal{A}})$.

Moreover, if $\Phi \in L_{l o c}^{p}(I \times \widetilde{\mathcal{A}})_{m v s}$, then we denote by

$$
L_{p}(\Phi) \equiv\left\{\phi \in L^{p}(\widetilde{\mathcal{A}}): \phi \text { is a selection of } \Phi\right\}
$$


Let $f, g \in L_{\gamma}^{2}(\mathbb{R})_{+}, \pi \in L_{B(\gamma), \text { loc }}^{\infty}\left(\mathbb{R}_{+}\right), \mathbb{I}$, the identity map on $\mathcal{R} \otimes \Gamma\left(L_{\gamma}^{2}\left(\mathbb{R}_{+}\right)\right)$, and $M$ is any of the stochastic processes $A_{f}, A_{g}^{+}, \Lambda_{\pi}$ and $s \mapsto s \mathbb{I}, s \in \mathbb{R}_{+}$.

We introduce the stochastic integral\{resp. differential\} expressions as follows:

If $\Phi \in L_{l o c}^{2}(I \times \widetilde{\mathcal{A}})_{m v s}$ and $(t, X) \in I \times L_{l o c}^{2}(\widetilde{\mathcal{A}})$, then

$$
\int_{t_{0}}^{t} \Phi(s, X(s)) d M(s) \equiv\left\{\int_{t_{0}}^{t} \phi(s) d M(s): \phi \in L_{2}(\Phi)\right\}
$$

This leads to the following definition:

Definition 2.3. Let $E, F, G, H \in L_{l o c}^{2}(I \times \widetilde{\mathcal{A}})$ and $\left(t_{0}, x_{0}\right)$ be a fixed point of $I \times \widetilde{\mathcal{A}}$. Then a relation of the form

$$
\begin{aligned}
d X(t) & \in E(t, X(t)) d \Lambda_{\pi}(t)+F(t, X(t)) d A_{f}(t) \\
& +G(t, X(t)) d A_{g}^{+}(t)+H(t, X(t)) d t \text { almost all } t \in I \\
X\left(t_{0}\right) & =x_{0}
\end{aligned}
$$

is called Quantum stochastic differential inclusions(QSDI) with coefficients $E$, $F, G, H$ and initial data $\left(t_{0}, x_{0}\right)$.

Equation(2.1) is understood in the integral form:

$$
\begin{array}{r}
X(t) \in x_{0}+\int_{t_{0}}^{t}\left(E(s, X(s)) d \Lambda_{\pi}(s)+F(s, X(s)) d A_{f}(s)\right. \\
\left.+G(s, X(s)) d A_{g}^{+}(s)+H(s, X(s)) d s\right), \quad t \in I
\end{array}
$$

called a stochastic integral inclusion with coefficients $E, F, G, H$ and initial data $\left(t_{0}, x_{0}\right)$

An equivalent form of (2.1) has been established in [7], Theorem 6.2 as follows:

For $\eta, \xi \in \mathbb{D} \underline{\otimes} \mathbb{E}, \alpha, \beta \in L_{\gamma}^{2}\left(\mathbb{R}_{+}\right)$with $\eta=c \otimes e(\alpha), \xi=d \otimes e(\beta)$, define the following complex-valued functions:

$$
\begin{aligned}
\mu_{\alpha \beta}, \nu_{\beta}, \sigma_{\alpha} & : I \rightarrow \mathbb{C}, \quad I \subset \mathbb{R}_{+}, \quad \text { by } \\
\mu_{\alpha \beta}(t) & =\langle\alpha(t), \pi(t) \beta(t)\rangle_{\gamma} \\
\nu_{\beta}(t) & =\langle f(t), \beta(t)\rangle_{\gamma} \\
\sigma_{\alpha}(t) & =\langle\alpha(t), g(t)\rangle_{\gamma}
\end{aligned}
$$

$t \in I, f, g \in L_{\gamma, l o c}^{2}\left(\mathbb{R}_{+}\right), \pi \in L_{B(\gamma), l o c}^{\infty}$. To these functions we associate the maps $\mu E, \nu F, \sigma G, \mathbb{P}$ from $I \times \widetilde{\mathcal{A}}$ into the set of sesquilinear forms on $\mathbb{D} \underline{\mathbb{E}}$ defined by 


$$
\begin{aligned}
&(\mu E)(t, x)(\eta, \xi)=\left\{\left\langle\eta, \mu_{\alpha \beta}(t) p(t, x) \xi\right\rangle: p(t, x) \in E(t, x)\right\} \\
&(\nu F)(t, x)(\eta, \xi)=\left\{\left\langle\eta, \nu_{\beta}(t) q(t, x) \xi\right\rangle: q(t, x) \in F(t, x)\right\} \\
&(\sigma G)(t, x)(\eta, \xi)=\left\{\left\langle\eta, \sigma_{\alpha}(t) u(t, x) \xi\right\rangle: u(t, x) \in G(t, x)\right\} \\
& \mathbb{P}(t, x)(\eta, \xi)=(\mu E)(t, x)(\eta, \xi)+(\nu F)(t, x)(\eta, \xi) \\
&+(\sigma G)(t, x)(\eta, \xi)+H(t, x)(\eta, \xi) \\
& H(t, x)(\eta, \xi)=\{v(t, x)(\eta, \xi): v(., X(.)) \\
&\text { is } \left.\text { a selection of } H(., X(.)) \forall X \in L_{l o c}^{2}(\widetilde{\mathcal{A}})\right\}
\end{aligned}
$$

Then Problem (2.1) is equivalent to

$$
\begin{aligned}
\frac{d}{d t}\langle\eta, X(t) \xi\rangle & \in \mathbb{P}(t, X(t))(\eta, \xi) \\
X\left(t_{0}\right) & =x_{0}
\end{aligned}
$$

for arbitrary $\eta, \xi \in \mathbb{D} \otimes \mathbb{E}$, almost all $t \in I$. Hence the existence of solution of (2.1) implies the existence of solution of (2.3) and vice-versa.

As explained in [7], for the sesquilinear form valued map $\mathbb{P}$ :

$$
\mathbb{P}(t, x)(\eta, \xi) \neq \widetilde{\mathbb{P}}(t,\langle\eta, x \xi\rangle)
$$

for some complex-valued multifunction $\widetilde{\mathbb{P}}$ defined on $I \times \mathbb{C}$ for $t \in I, x \in \widetilde{\mathcal{A}}$, $\eta, \xi \in \mathbb{D} \underline{\otimes} \mathbb{E}$.

The notion of solution of (2.1) or equivalently (2.3) is defined as follows:

Definition 2.4. By a solution of (2.1) or equivalently (2.3), we mean a stochastic process $\varphi \in \operatorname{Ad}(\widetilde{\mathcal{A}})_{w a c} \cap L_{\text {loc }}^{2}(\widetilde{\mathcal{A}})$ such that

$$
\begin{aligned}
d \varphi(t) & \in E(t, \varphi(t)) d \Lambda_{\pi}(t)+F(t, \varphi(t)) d A_{f}(t) \\
& +G(t, \varphi(t)) d A_{g}^{+}(t)+H(t, \varphi(t)) d t \quad \text { almost all } t \in I \\
\varphi\left(t_{0}\right) & =\varphi_{0}
\end{aligned}
$$

or equivalently

$$
\begin{aligned}
\frac{d}{d t}\langle\eta, \varphi(t) \xi\rangle & \in \mathbb{P}(t, \varphi(t))(\eta, \xi) \\
\varphi\left(t_{0}\right) & =\varphi_{0}
\end{aligned}
$$

for arbitrary $\eta, \xi \in \mathbb{D} \underline{\otimes} \mathbb{E}$, almost all $t \in I$. 


\subsection{Upper Semicontinuous Multivalued Maps}

Let $\mathcal{N} \subseteq \widetilde{\mathcal{A}}$ and $I \subseteq \mathbb{R}_{+}$. For arbitrary $\eta, \xi \in \mathbb{D} \underline{\otimes} \mathbb{E},(t, x),\left(t_{0}, x_{0}\right) \in I \times \mathcal{N}$ and $\epsilon, \delta_{\eta \xi}>0$ we define $d_{\eta \xi}$ as:

$$
d_{\eta \xi}\left((t, x),\left(t_{0}, x_{0}\right)\right)=\max \left\{\left|t-t_{0}\right|,\left\|x-x_{0}\right\|_{\eta \xi}\right\}
$$

The following sets shall be defined as applicable in the sequel:

$$
\begin{aligned}
B_{\eta \xi, \epsilon}(0) & =\left\{x \in \widetilde{\mathcal{A}}:\|x\|_{\eta \xi}<\epsilon\right\} \\
B_{\delta_{\eta \xi}}\left(t_{0}, x_{0}\right) & =\left\{(t, x) \in I \times \mathcal{N}: d_{\eta \xi}\left((t, x),\left(t_{0}, x_{0}\right)\right)<\delta_{\eta \xi}\right\}
\end{aligned}
$$

Furthermore, for a sesquilinear form valued map; $\Phi: I \times \mathcal{N} \rightarrow 2^{\operatorname{sesq}(\mathbb{D} \otimes \underline{\mathbb{E}})^{2}}$, we define

$$
B_{\Phi, \epsilon}(0)=\{\varphi(t, x)(\eta, \xi) \in \Phi(t, x)(\eta, \xi):|\varphi(t, x)(\eta, \xi)|<\epsilon\}
$$

In what follows we shall present the definition of upper semicontinuous multivalued maps:

Definition 2.5. A map $\Phi: I \times \mathcal{N} \rightarrow \operatorname{clos}(\widetilde{\mathcal{A}})$ will be said to be upper semicontinuous at a point $\left(t_{0}, x_{0}\right) \in I \times \mathcal{N}$ if for every $\eta, \xi \in \mathbb{D} \underline{\otimes} \mathbb{E}, \epsilon>0$, there exists $\delta_{\eta \xi}=\delta_{\eta \xi}\left(\left(t_{0}, x_{0}\right), \epsilon\right)>0$ such that $\Phi(t, x) \subset \Phi\left(t_{0}, x_{0}\right)+B_{\eta \xi, \epsilon}(0)$ on $B_{\delta_{\eta \xi}}\left(t_{0}, x_{0}\right)$.

The map $\Phi$ will be said to be upper semicontinuous on $I \times \mathcal{N}$, if it upper semicontinuous at every point $(t, x) \in I \times \mathcal{N}$;

For a sesquilinear form valued multifunction $\mathbb{P}$

Definition 2.6. A map $\mathbb{P}: I \times \mathcal{N} \rightarrow 2^{\operatorname{sesq}(\mathbb{D} \underline{\otimes} \mathbb{E})^{2}}$ will be said to be upper semicontinuous at a point $\left(t_{0}, x_{0}\right) \in I \times \mathcal{N}$ if for every $\eta, \xi \in \mathbb{D} \underline{\otimes} \mathbb{E}, \epsilon>0$, there exists $\delta_{\eta \xi}=\delta_{\eta \xi}\left(\left(t_{0}, x_{0}\right), \epsilon\right)>0$ such that

$$
\mathbb{P}(t, x)(\eta, \xi) \subset \mathbb{P}\left(t_{0}, x_{0}\right)(\eta, \xi)+B_{\mathbb{P}, \epsilon}(0)
$$

on $B_{\delta_{\eta \xi}}\left(t_{0}, x_{0}\right)$.

$\mathbb{P}$ is said to be upper semicontinuous on $I \times \mathcal{N}$, if it is upper semicontinuous at every point $(t, x) \in I \times \mathcal{N}$.

The following proposition shows that if the maps $\mu E, \nu F, \sigma G$ and $H$ defined in (2.2) are upper semicontinuous on $I \times \mathcal{N}$ then the map $\mathbb{P}$ given by:

$$
\begin{aligned}
\mathbb{P}(t, x)(\eta, \xi) & =(\mu E)(t, x)(\eta, \xi)+(\nu F)(t, x)(\eta, \xi) \\
& +(\sigma G)(t, x)(\eta, \xi)+H(t, x)(\eta, \xi)
\end{aligned}
$$

is upper semicontinuous on $I \times \mathcal{N}$. 
Proposition 2.1. : Suppose $E, F, G, H$ lie $L_{l o c}^{2}(I \times \widetilde{\mathcal{A}})_{m v s}, \mathcal{N} \in \operatorname{clos}(\widetilde{\mathcal{A}})$, non-empty and $\Phi: I \times \mathcal{N} \rightarrow 2^{\text {sesq. }(\mathbb{D} \underline{\otimes} \mathbb{E})^{2}} ; \Phi \in\{\mu E, \nu F, \sigma G, H\}$.

If for every $\eta, \xi \in \mathbb{D} \otimes \mathbb{E}$, the maps, $\Phi(.,).(\eta, \xi)$ are upper semicontinuous on $I \times \mathcal{N}$, then $\mathbb{P}$ is upper semicontinuous on $I \times \mathcal{N}$.

Proof. Given that $\mu E(. .,).(\eta, \xi), \nu F(.,).(\eta, \xi), \sigma G(. .).(\eta, \xi), H(.,).(\eta, \xi)$ are upper semicontinuous on $I \times \mathcal{N}$ implies that they are upper semicontinuous at every point $\left(t^{\prime}, x^{\prime}\right) \in I \times \mathcal{N}$; that is for every $\eta, \xi \in \mathbb{D} \otimes \mathbb{E}, \epsilon>0$, there exists $\delta_{\eta \xi}^{\Phi}=\delta_{\eta \xi}^{\Phi}\left(\left(t^{\prime}, x^{\prime}\right), \epsilon\right)>0, \Phi \in\{\mu E, \nu F, \sigma G, H\}$ such that the following relations hold;

$$
\Phi(t, x)(\eta, \xi) \subset \Phi\left(t^{\prime}, x^{\prime}\right)(\eta, \xi)+B_{\Phi, \epsilon}(0) \text { on } B_{\delta_{\eta \xi}^{\Phi}}\left(t^{\prime}, x^{\prime}\right)
$$

But for each $\Phi \in\{\mu E, \nu F, \sigma G, H\}, B_{\Phi, \epsilon}(0) \subset B_{\mathbb{P}, \epsilon}(0)$.

Therefore for each $\Phi \in\{\mu E, \nu F, \sigma G, H\}$,

$$
\Phi(t, x)(\eta, \xi) \subset \Phi\left(t^{\prime}, x^{\prime}\right)(\eta, \xi)+B_{\mathbb{P}, \epsilon}(0) \text { on } B_{\delta_{\eta \xi}^{\Phi}}\left(t^{\prime}, x^{\prime}\right)
$$

then there exists $\delta_{\eta \xi}=\delta_{\eta \xi}\left(\left(t^{\prime}, x^{\prime}\right), \epsilon\right)>0$ such that

$$
\begin{aligned}
& (\mu E)(t, x)(\eta, \xi)+(\nu F)(t, x)(\eta, \xi)+(\sigma G)(t, x)(\eta, \xi)+H(t, x)(\eta, \xi) \\
& \quad \subset\left[(\mu E)\left(t^{\prime}, x^{\prime}\right)(\eta, \xi)+(\nu F)\left(t^{\prime}, x^{\prime}\right)(\eta, \xi)+(\sigma G)\left(t^{\prime}, x^{\prime}\right)(\eta, \xi)\right. \\
& \left.\quad+H\left(t^{\prime}, x^{\prime}\right)(\eta, \xi)\right]+B_{P_{\eta \xi}, \epsilon}(0)
\end{aligned}
$$

on $B_{\delta_{\eta \xi}}\left(t^{\prime}, x^{\prime}\right)$

where

$$
\delta_{\eta \xi}=\min \left\{\delta_{\eta \xi}^{\Phi}\right\}>0 ; \Phi \in\{\mu E, \nu F, \sigma G, H\}
$$

that is,

$$
\mathbb{P}(t, x)(\eta, \xi) \subset \mathbb{P}\left(t^{\prime}, x^{\prime}\right)(\eta, \xi)+B_{\mathbb{P}, \epsilon}(0)
$$

then $\mathbb{P}$ is upper semicontinuous at $\left(t^{\prime}, x^{\prime}\right)$. Since $\left(t^{\prime}, x^{\prime}\right)$ was arbitrarily chosen in $I \times \mathcal{N}$, we conclude that $\mathbb{P}(.,).(\eta, \xi)$ is upper semicontinuous on $I \times \mathcal{N}$. 


\section{Main Results}

In this section we establish an approximate selection theorem for upper semicontinuous multivalued maps as it applies to our non commutative setting. We then establish the existence of solutions of quantum stochastic differential inclusions with upper semicontinuous coefficients having minimal selections contained in some compact subsets of $\mathbb{C}$.

For an arbitrary $\eta, \xi \in \mathbb{D} \underline{\otimes} \mathbb{E}$, we define

$$
\widetilde{\mathcal{A}}(\eta, \xi)=\{\langle\eta, x \xi\rangle: x \in \widetilde{\mathcal{A}}\} .
$$

The following Lemma is an adaptation of Mean value theorem ([1], Theorem $0.5 .3)$

Lemma 3.1. Let $(a, b) \subset \mathbb{R}$ be an interval and $\eta, \xi \in \mathbb{D} \underline{\otimes} \mathbb{E}$.

Suppose a map $v_{\eta \xi}:[a, b] \rightarrow \widetilde{\mathcal{A}}(\eta, \xi)$, is such that

$$
\begin{array}{r}
v_{\eta \xi}(t) \in K_{\eta \xi} \subset \widetilde{\mathcal{A}}(\eta, \xi), \quad \forall t \in(a, b) \text { and } v_{\eta \xi} \in L_{l o c}^{1}(a, b ; \widetilde{\mathcal{A}}(\eta, \xi)), \\
v_{\eta \xi}(t)=\langle\eta, v(t) \xi\rangle, \quad v(t) \in L_{l o c}^{1}(a, b ; \widetilde{\mathcal{A}})
\end{array}
$$

Then $\forall, t_{0}, t_{1} \in(a, b)$

$$
\int_{t_{0}}^{t_{1}} v_{\eta \xi}(s) d s \in\left(t_{1}-t_{0}\right) \overline{c o}\left(K_{\eta \xi}\right) .
$$

Proof. Since the map $v_{\eta \xi} \in L_{l o c}^{1}(a, b ; \widetilde{\mathcal{A}}(\eta, \xi))$, then $v_{\eta \xi}($.$) can be approxi-$ mated in $L_{l o c}^{1}$ by a sequence of simple functions $v_{\eta \xi, n}()=.\sum_{i} y_{n, i} \chi_{E_{i}}().(\eta, \xi)$.

Where $\chi_{E_{i}}(\eta, \xi)$ denotes the characteristic function of a measurable set $E_{i}(\eta, \xi) \in K_{\eta \xi}$ and $y_{n, i} \in \mathbb{C}$.

Fix $t_{1}$ and $t_{0}$ in $[a, b]$. Then

$$
\int_{t_{0}}^{t_{1}} v_{\eta \xi, n}(s) d s \in\left(t_{1}-t_{0}\right) \operatorname{co}\left(K_{\eta \xi}\right)
$$

By letting $n$ go to infinity we have,

$$
\int_{t_{0}}^{t_{1}} v_{\eta \xi}(s) d s \in\left(t_{1}-t_{0}\right) \overline{c o}\left(K_{\eta \xi}\right)
$$


Let $K_{\eta \xi}$ be a closed convex subset of $\widetilde{\mathcal{A}}(\eta, \xi)$. We associate to any $x \in \mathbb{C}$ a unique element $\pi_{K_{\eta \xi}}(x) \in K_{\eta \xi}$ satisfying

$$
\left|x-\pi_{K_{\eta \xi}}(x)\right|=\min _{y \in K_{\eta \xi}}|x-y|
$$

It is characterized by the following variational inequality

$$
\left\langle\pi_{K_{\eta \xi}}(x)-x, \pi_{K_{\eta \xi}}(y)-y\right\rangle \leq 0 \quad \forall y \in K_{\eta \xi}
$$

The map $\pi_{K_{\eta \xi}}: \mathbb{C} \rightarrow K_{\eta \xi}$ is called the projector (of best approximation) onto $K_{\eta \xi}$. If $K_{\eta \xi}$ is a convex subset, we set

$$
m\left(K_{\eta \xi}\right)=\pi_{K_{\eta \xi}}(0)
$$

which is the element of $K_{\eta \xi}$ with minimal modulus in $\mathbb{C}$.

Definition 3.1. Let $M \subset \widetilde{\mathcal{A}}$ be non-empty and $\varphi: M \rightarrow \operatorname{ses} q(\mathbb{D} \otimes \mathbb{E})^{2}$. For an arbitrary pair $\eta, \xi \in \mathbb{D} \otimes \mathbb{E}$;

(i) $\varphi$ is said to be locally compact if for each point $x \in M$ there exists a neighbourhood $N(x)$ such that $\varphi(N(x))(\eta, \xi)$ is a compact subset of $\mathbb{C}$.

(ii) $\varphi$ is said to be locally equicompact if for any $x \in M$, there exists a compact subset $K_{\eta \xi} \subset \mathbb{C}$, a neighbourhood $N(x)$ of $x$ and $\epsilon_{0}>0$, such that for every $\epsilon<\epsilon_{0}, \varphi_{\epsilon}(N(x))(\eta, \xi) \subset K_{\eta \xi}$.

\subsection{Locally Lipschitzian Partition of Unity}

Definition 3.2. (a) Let $M \subset \widetilde{\mathcal{A}}$; for an arbitrary pair $\eta, \xi \in \mathbb{D} \underline{\otimes} \mathbb{E}$, a map $\varphi: M \rightarrow \operatorname{sesq} .(\mathbb{D} \underline{\mathbb{E}})^{2}$ is said to be locally Lipschitzian if for every $x \in M$ there exists a neighbourhood $V(x)$ such that $\forall x_{1}, x_{2} \in V(x)$, there exists $L_{\eta \xi}>0$, such that

$$
\left|\phi\left(x_{1}\right)(\eta, \xi)-\phi\left(x_{2}\right)(\eta, \xi)\right|<L_{\eta \xi}\left\|x_{1}-x_{2}\right\|_{\eta \xi}
$$

(b) Let $M \subset \widetilde{\mathcal{A}}$; A family of open subsets of $M,\left\{\Omega_{i}\right\}_{i \in I}$, such that $M=\cup_{i \in I} \Omega_{i}$ is called an open covering of $M$.

Let $\left\{\Omega_{i}\right\}_{i \in I}$ and $\left\{\omega_{j}\right\}_{j \in J}$ be two coverings of $M .\left\{\Omega_{i}\right\}_{i \in I}$ is a refinement of $\left\{\omega_{j}\right\}_{j \in J}$ if for every $i \in I$, there exists $j \in J$ such that $\Omega_{i} \subset \omega_{j}$. Let $\left\{\Omega_{i}\right\}_{i \in I}$ be a covering, if $J \subset I$ and $\left\{\Omega_{j}\right\}_{j \in J}$ is again a covering, it is called a subcovering.

(c) A covering $\left\{\Omega_{i}\right\}_{i \in I}$ of $M$ is called locally finite if for every $x \in M$, there exists a neighbourhood $V$ of $x$ such that $\Omega_{i} \cap V \neq \emptyset$ only for a finite number of indexes.

(d) Let $\varphi: M \rightarrow \operatorname{ses} q .(\mathbb{D} \underline{\mathbb{E}})^{2}$, the closure of $\{x \in M: \varphi(x)(\eta, \xi) \neq 0\}$ is called the support of $\varphi,(\operatorname{supp}(\varphi))$. 
A family $\left\{\varphi_{i}\right\}_{i \in I}$ is called a locally Lipschitzian partition of unity if for all $i \in I$;

(i) $\varphi_{i}$ is locally Lipschitzian and non negative

(ii) the supports of $\varphi_{i}$ are closed locally finite covering of $M$;

(iii) for each $x \in M, \sum_{i \in I} \varphi_{i}(x)=1$.

We say that a partition of unity $\left\{\varphi_{i}\right\}_{i \in I}$ is subordinated to a covering $\left\{\Omega_{i}\right\}_{i \in I}$ if $\forall i \in I, \operatorname{supp}\left(\varphi_{i}\right) \subset \Omega_{i}$.

In what follows we shall establish the existence of locally Lipschitzian partition of unity.

Proposition 3.1. Let $M \subset \widetilde{\mathcal{A}}$ be a non-empty compact subset of $\widetilde{\mathcal{A}}$. To any locally finite open covering $\left\{\Omega_{i}\right\}_{i \in I}$ of $M$, there exists a locally Lipschitzian partition of unity subordinate to $\left\{\Omega_{i}\right\}_{i \in I}$

Proof. There exists an open covering $\left(\omega_{i}\right)_{i \in I}$ of $M$ such that $\overline{\omega_{i}} \subset \Omega_{i}$ for any $i \in I$, since $M$ is compact. Also, since $\left(\Omega_{i}\right)_{i \in I}$ is locally finite, $\left\{\omega_{i}\right\}_{i \in I}$ is also locally finite.

For any $i \in I$, we define $\varphi_{i}: M \rightarrow \mathbb{R}_{+}$by

$$
\varphi_{i}(x)=d_{\eta \xi}\left(x, M \backslash \omega_{i}\right)
$$

where $d_{\eta \xi}\left(., M \backslash \omega_{i}\right)$ denotes the distance function to the subset $M \backslash \omega_{i}=\omega_{i}^{c}$.

Each $\varphi_{i}$ is Lipschitzian and $\operatorname{supp}\left(\varphi_{i}\right)=\overline{\left\{x \in M: \varphi_{i}(x) \neq 0\right\}}=\overline{\omega_{i}} \subset \Omega_{i}$

For any $i \in I$, we define the function $\psi_{i}: M \rightarrow \mathbb{R}_{+}$such that

$$
\psi_{i}(x)=\frac{\varphi_{i}(x)}{\sum_{j \in I} \varphi_{j}(x)} \text { for any } x \in M
$$

For any $x \in M, \sum_{j \in I} \varphi_{j}(x)$ is well defined since the covering $\left(\omega_{i}\right)_{i \in I}$ is locally finite.

Also, $\sum_{j \in I} \varphi_{j}(x)>0$, since for at least one index $j \in I, x \in \omega_{j}, \varphi_{j}(x)=$ $d_{\eta \xi}\left(x, M \backslash \omega_{j}\right)>0$.

Furthermore each $\psi_{i}$ is continuous on $M$ and takes its values in the interval $[0,1]$.

Moreover, $\operatorname{supp}_{i}=\operatorname{supp}_{i}=\overline{\omega_{i}} \subset \Omega_{i}$ and

$$
\sum_{i \in I} \psi_{i}(x)=1 \text { for any } x \in M
$$

We now prove that each function $\psi_{i}$ is locally Lipschitzian on $M$. 
Let $x \in M$ be given, then there exists a neighbourhood $V(x)$ of $x$ which meets only a finite number of $\omega_{i}, i \in I$, say

$$
\omega_{i_{1}}, \omega_{i_{2}}, \ldots, \omega_{i_{p}}
$$

When $y \in V(x), \psi_{i}(y)=0$, if $i \notin\left\{i_{1}, i_{2}, \ldots, i_{p}\right\}$

Consider $\psi_{i_{k}}$ with $i_{k} \in\left\{i_{1}, i_{2}, \ldots, i_{p}\right\}$. Since

$$
\sum_{i \in I} \varphi_{i}(x)=\sum_{i \in\left\{i_{1}, i_{2}, \ldots, i_{p}\right\}} \varphi_{i}(x)>0
$$

by continuity, there exists a neighbourhood $W(x) \subset V(x)$ of $x$ and $m>0$, $M>0$ such that:

$$
m \leq \sum_{i \in I} \varphi_{i}(y)=\sum_{i \in \in\left\{i_{1}, i_{2}, \ldots, i_{p}\right\}} \varphi_{i}(y) \leq M \text { for any } y \in W(x)
$$

Then for any $y, z \in W(x)$ we get:

$$
\begin{aligned}
\left|\psi_{i_{k}}(y)-\psi_{i_{k}}(z)\right| & =\left|\frac{\varphi_{i_{k}}(y)}{\sum_{i \in\left\{i_{1}, \ldots, i_{p}\right\}} \varphi_{i}(y)}-\frac{\varphi_{i_{k}}(z)}{\sum_{i \in\left\{i_{1}, \ldots, i_{p}\right\}} \varphi_{i}(z)}\right| \\
& =\frac{\left|\varphi_{i_{k}}(y) \sum_{i \in\left\{i_{1}, \ldots, i_{p}\right\}} \varphi_{i}(z)-\varphi_{i_{k}}(z) \sum_{i \in\left\{i_{1}, \ldots, i_{p}\right\}} \varphi_{i}(y)\right|}{\sum_{i \in\left\{i_{1}, \ldots, i_{p}\right\}} \varphi_{i}(y) \sum_{i \in\left\{i_{1}, \ldots, i_{p}\right\}} \varphi_{i}(z)} \\
& \leq \frac{\left|\varphi_{i_{k}}(y) \sum_{i \in\left\{i_{1}, \ldots, i_{p}\right\}} \varphi_{i}(z)-\varphi_{i_{k}}(z) \sum_{i \in\left\{i_{1}, \ldots, i_{p}\right\}} \varphi_{i}(y)\right|}{m^{2}} \\
& \leq \frac{1}{m^{2}} \sum_{i \in\left\{i_{1}, \ldots, i_{p}\right\}}\left|\varphi_{i_{k}}(y) \varphi_{i}(z)-\varphi_{i_{k}}(z) \varphi_{i}(y)\right| \\
& \leq \frac{1}{m^{2}} \sum_{i \in\left\{i_{1}, \ldots, i_{p}\right\}}\left|\varphi_{i}(z) \varphi_{i_{k}}(z)-\varphi_{i_{k}}(z) \varphi_{i}(y)\right| \\
& +\frac{1}{m^{2}} \sum_{i \in\left\{i_{1}, \ldots, i_{p}\right\}}\left|\varphi_{i}(z) \varphi_{i_{k}}(y)-\varphi_{i}(z) \varphi_{i_{k}}(z)\right| \\
& \leq \frac{1}{m^{2}} \sum_{i \in\left\{i_{1}, \ldots, i_{p}\right\}}\left|\varphi_{i_{k}}(z)\right| \cdot\left|\varphi_{i}(z)-\varphi_{i}(y)\right| \\
& +\frac{1}{m^{2}} \sum_{i \in\left\{i_{1}, \ldots, i_{p}\right\}}\left|\varphi_{i}(z)\right| \cdot\left|\varphi_{i_{k}}(y)-\varphi_{i_{k}}(z)\right| \\
& \leq \frac{2 M p}{m^{2}}\|y-z\| \eta \xi
\end{aligned}
$$


Since each $\varphi_{i}$ for $i \in\left\{i_{1}, \ldots, i_{p}\right\}$ is bounded above by $M$ on $W(x)$ and Lipschitzian with constant equal to 1 . Hence $\psi_{i_{k}}$ with $i_{k} \in\left\{i_{1}, \ldots, i_{p}\right\}$ is Lipschitzian on $W(x)$ with the Lipschitz constant $\frac{2 M p}{m^{2}}$.

\subsection{An Approximate Selection Theorem}

Let $M \subset \widetilde{\mathcal{A}}$, and $\Phi: M \rightarrow 2^{\text {sesq }(\mathbb{D} \underline{\otimes} \mathbb{E})^{2}}, \epsilon>0$, we define the ball $B_{\epsilon}^{\Phi}$, a subset of the graph of $\Phi$, as:

$$
B_{\epsilon}^{\Phi}=\left\{(x, y) \in M \times \mathbb{C}: y \in \Phi(x)(\eta, \xi), \max \left\{\|x\|_{\eta \xi},|y|\right\}<\epsilon\right\}
$$

The following lemma is a noncommutative analogue of approximate selection theorem in Aubin and Cellina[1].

Lemma 3.2. (Approximate selection theorem): Let $M \subset \widetilde{\mathcal{A}}$ be nonempty and compact subset of $\widetilde{\mathcal{A}}$; and $\Phi: M \rightarrow 2^{\text {sesq }(\mathbb{D} \otimes \mathbb{E})^{2}}$, such that for any pair $\eta, \xi \in \mathbb{D} \underline{\otimes} \mathbb{E}, x \in M$,

$$
x \rightarrow \Phi(x)(\eta, \xi)
$$

is upper semicontinuous and convex-valued.

Then, for every $\epsilon>0$, there exists a map

$$
\varphi_{\epsilon}: M \rightarrow \operatorname{sesq}(\mathbb{D} \underline{\otimes} \mathbb{E})^{2}
$$

such that $x \rightarrow \varphi_{\epsilon}(x)(\eta, \xi)$ is locally Lipschitzian, $\varphi_{\epsilon}(M)(\eta, \xi) \subseteq \operatorname{co}(\Phi(M)(\eta, \xi))$ and

$$
\operatorname{Graph}\left(\varphi_{\epsilon}(.)(\eta, \xi)\right) \subset \operatorname{Graph}(\Phi(.)(\eta, \xi))+B_{\epsilon}^{\Phi}
$$

If the minimal selection is locally compact, the family $\left\{\varphi_{\epsilon}\right\}$ is locally equicompact.

Proof. (a) Fix $\epsilon>0$. For every $x \in M$, there exists $\delta(x)$ such that for any $x * \in B_{\eta \xi, \delta(x)}(x)$, we have $\Phi(x *) \subset \Phi(x)+B_{\eta \xi, \frac{\epsilon}{2}}$. We can take $\delta(x)<\frac{\epsilon}{2}$. The family $\left\{B_{\eta \xi, \frac{\delta(x)}{4}}(x)\right\}_{x \in M}$ covers $M$.

Let $\left\{\mathcal{U}_{i}\right\}_{i \in I}$ be a locally finite refinement and $\left\{a_{i}().\right\}$ a locally Lipschitzian partition of unity subordinate to it. Choose for each $i$ an $\bar{x}_{i}$ and define $\varphi_{\epsilon}().(\eta, \xi)$ to be

$$
\varphi_{\epsilon}(x)(\eta, \xi)=\sum a_{i} m\left(\Phi\left(\bar{x}_{i}\right)(\eta, \xi)\right)
$$

$\varphi_{\epsilon}(\eta, \xi)$ is well defined and locally Lipschitzian and its range is in the convex hull of the range of $\Phi$.

Fix $x \in M$. Then $a_{i}(x)$ is strictly positive only for a finite subset $I_{x} \in I$. For $i \in I_{x}$, set $x_{i}$ to be such that $\mathcal{U}_{i} \subset B_{\eta \xi, \frac{\delta\left(x_{i}\right)}{4}}\left(x_{i}\right)$. Set $\delta_{i}=\delta\left(x_{i}\right)$ and let $j \in I_{x}$ 
be such that $\delta_{j}=\max _{i \in I_{x}} \delta_{i}$. Then $x_{i} \in B_{\eta \xi, \frac{\delta_{j}}{2}}\left(x_{j}\right)$ and thus $\mathcal{U}_{i} \subset B_{\eta \xi, \delta_{j}}\left(x_{j}\right)$. Therefore, for any $i \in I_{x}$,

$$
m\left(\Phi\left(x_{i}\right)(\eta, \xi)\right) \in \Phi\left(\mathcal{U}_{i}\right)(\eta, \xi) \subset \Phi\left(B_{\eta \xi, \delta_{j}}\right)\left(x_{j}\right)(\eta, \xi) \subset \Phi\left(x_{j}\right)(\eta, \xi)+B_{\Phi, \frac{\epsilon}{2}}
$$

Since the latter set is convex, we deduce that $\varphi_{\epsilon}(x)(\eta, \xi) \in \Phi\left(x_{j}\right)(\eta, \xi)+B_{\Phi, \frac{\epsilon}{2}}$. So, there exists $y_{j} \in \Phi\left(x_{j}\right)(\eta, \xi)$ such that $\left|\varphi_{\epsilon}(x)(\eta, \xi)-y_{j}\right| \leq \frac{\epsilon}{2}$. Then

$$
\begin{aligned}
& d\left(\left(x, \varphi_{\epsilon}(x)(\eta, \xi)\right),\left(x_{j}, y_{j}\right)\right) \leq\left\|x-x_{j}\right\|_{\eta \xi}+\left|\varphi_{\epsilon}(x)(\eta, \xi)-y_{j}\right| \leq \epsilon, \\
& \text { i.e. }\left(x, \varphi_{\epsilon}(x)(\eta, \xi)\right) \in \operatorname{graph}(\Phi)+B_{\epsilon}^{\Phi}
\end{aligned}
$$

(b) Let us prove that the family $\left\{\varphi_{\epsilon}\right\}$ is equicompact. Fix $x \in M$. There exist $\omega>0$ and a compact convex subset $K_{\eta \xi}$ such that for any $x * \in B_{\eta \xi, \omega}(x)$ we have $m(\Phi(x *)(\eta, \xi)) \in K_{\eta \xi}$.

Set $\epsilon_{0}=\frac{\omega}{4}$ and $N(x)$ to be $B_{\eta \xi}, \frac{\omega}{2}(x)$. For any $\epsilon<\epsilon_{0}$, the above construction shows that we can associate with any $x^{*} \in N(x)$ a point $\bar{x} \in B_{\eta \xi, \epsilon}(x *)$ and $\varphi_{\epsilon}(x *)(\eta, \xi)$, which is a convex combination of images through $m(\varphi().(\eta, \xi))$ of points in $B_{\eta \xi, \epsilon}(\bar{x}) \subset B_{\eta \xi, \omega}(x)$. These images are in $K_{\eta \xi}$ and so is their convex combination.

\subsection{Existence Results}

Lemma 3.3. : Suppose that the multivalued sesquilinear form:

$\mathbb{P}: I \times \widetilde{\mathcal{A}} \rightarrow 2^{\text {sesq }(\mathbb{D} \otimes \mathbb{E})^{2}}$ is upper semicontinuous with compact and convex values in $\mathbb{C}$. A stochastic process $\varphi \in \operatorname{Ad}(\widetilde{\mathcal{A}})_{\text {wac }} \cap L_{l o c}^{2}(\widetilde{\mathcal{A}})$ is a solution to the inclusion

$$
\begin{array}{r}
\frac{d}{d t}\langle\eta, X(t) \xi\rangle \in \mathbb{P}(t, X)(\eta, \xi) \\
X\left(t_{0}\right)=x_{0}
\end{array}
$$

if and only if for every pair $\left(t_{1}, t_{2}\right) \in I, \eta, \xi \in \mathbb{D} \underline{\otimes} \mathbb{E}$;

$$
\left\langle\eta, \varphi\left(t_{2}\right) \xi\right\rangle \in\left\langle\eta, \varphi\left(t_{1}\right) \xi\right\rangle+\int_{t_{1}}^{t_{2}} \mathbb{P}(s, \varphi(s))(\eta, \xi) d s
$$

Proof. Suppose that for every pair $\left(t_{1}, t_{2}\right) \in I, \eta, \xi \in \mathbb{D} \underline{\mathbb{E}}$

$$
\left\langle\eta, \varphi\left(t_{2}\right) \xi\right\rangle \in\left\langle\eta, \varphi\left(t_{1}\right) \xi\right\rangle+\int_{t_{1}}^{t_{2}} \mathbb{P}(s, \varphi(s))(\eta, \xi) d s
$$

Since $\mathbb{P}$ is compact-valued, there exists a compact set $K_{\eta \xi} \subset \mathbb{C}$ 
such that $\mathbb{P}(t, X)(\eta, \xi) \subset K_{\eta \xi}$, then from Lemma 8,

$$
\int_{t_{1}}^{t_{2}} \mathbb{P}(s, \varphi(s))(\eta, \xi) d s \subset \overline{c o}\left(K_{\eta \xi}\right)\left(t_{2}-t_{1}\right)
$$

We remark that

$$
\int_{t_{1}}^{t_{2}} \overline{c o}\left(K_{\eta \xi}\right) d s=\overline{c o}\left(K_{\eta \xi}\right)\left(t_{2}-t_{1}\right)
$$

From (3.2), (3.3) and (3.4), we have

$$
\left|\left\langle\eta, \varphi\left(t_{2}\right) \xi\right\rangle-\left\langle\eta, \varphi\left(t_{1}\right) \xi\right\rangle\right|<\|\mathbb{P}\|_{\eta \xi}\left(t_{2}-t_{1}\right)
$$

where

$$
\|\mathbb{P}\|_{\eta \xi}=\sup _{t, x \in I \times \widetilde{\mathcal{A}}} \rho(\mathbb{P}(t, x)(\eta, \xi), 0)
$$

Which implies that $\varphi$ is Lipschitzian, hence it is differentiable almost everywhere on $I$.

Let $t$ be a point where $\frac{d}{d t}\langle\eta, \varphi(t) \xi\rangle$ exists. Fix $\epsilon>0$, and let $\delta>0$ be such that $|t-\bar{t}| \leq \delta$ implies

$$
\mathbb{P}(\bar{t}, \varphi(\bar{t}))(\eta, \xi) \subset \mathbb{P}(t, \varphi(t))(\eta, \xi)+B_{\mathbb{P}, \epsilon}
$$

Then

$$
\begin{aligned}
\frac{d}{d t}\left\langle\eta, \varphi\left(t_{1}\right) \xi\right\rangle-\frac{d}{d t}\langle\eta, \varphi(t) \xi\rangle & \in \int_{t}^{t_{1}} \mathbb{P}(s, \varphi(s))(\eta, \xi) d s \\
& \subset \int_{t}^{t_{1}}\left[\mathbb{P}(s, \varphi(s))(\eta, \xi)+B_{\mathbb{P}, \epsilon}\right] d s \\
& =\left(t_{1}-t\right)\left[\mathbb{P}(t, \varphi(t))(\eta, \xi)+\epsilon B_{\eta \xi}\right] \quad \text { i.e. } \\
& \frac{d}{d t}\langle\eta, \varphi(t) \xi\rangle \in \mathbb{P}(t, \varphi(t))(\eta, \xi)+B_{\mathbb{P}, \epsilon}
\end{aligned}
$$

Since $\epsilon$ is arbitrarily chosen and $\mathbb{P}$ is closed then $\frac{d}{d t}\langle\eta, \varphi(t) \xi\rangle \in \mathbb{P}(t, \varphi(t))(\eta, \xi)$.

Conversely, suppose that $\varphi$ is a solution of quantum stochastic differential inclusion (4) on $I$, its derivative is a measurable selection of $\mathbb{P}(t, \varphi(t))$, hence we have

$$
\left\langle\eta, \varphi\left(t_{1}\right) \xi\right\rangle \in\left\langle\eta, \varphi\left(t_{2}\right) \xi\right\rangle+\int_{t_{1}}^{t_{2}} \mathbb{P}(s, \varphi(s))(\eta, \xi) d s
$$


Theorem 3.1. : Given a quantum stochastic differential inclusion (3.1) such that $\mathbb{P}: I \times \widetilde{\mathcal{A}} \rightarrow 2^{\text {sesq }(\mathbb{D} \underline{\otimes} \mathbb{E})^{2}}$ satisfies the following properties :

(i) $\mathbb{P}$ is a non-empty upper semicontinuous closed and convex valued map

(ii) There exists a compact set $K_{\eta \xi} \subset \mathbb{C}$ such that the map $(t, X) \rightarrow$ $m(\mathbb{P}(t, x)(\eta, \xi))$ remains in $K_{\eta \xi}$

Then for any $x_{0} \in \widetilde{\mathcal{A}}$ there exists an adapted weakly absolutely continuous function defined on $I$, a solution of (3.1)

Proof. For an arbitrary $\eta, \xi \in \mathbb{D} \otimes \mathbb{E}$, let $\left\{f_{n, \eta \xi}\right\}$ be a sequence of continuous single-valued maps approaching $\mathbb{P}$ in the sense of the approximate selection, where $f_{n, \eta \xi}(t, x(t)) \equiv f_{n}(t, x(t))(\eta, \xi)$. Let $T$ be arbitrarily chosen such that for $x_{n} \in \operatorname{Ad}(\widetilde{\mathcal{A}})_{w a c}, x_{n}:[0, T] \rightarrow \widetilde{\mathcal{A}}$, are solutions of

$$
\frac{d}{d t}\left\langle\eta, x_{n}(t) \xi\right\rangle=f_{n}\left(t, x_{n}(t)\right)(\eta, \xi), \quad x_{n}(0)=x_{0}
$$

Each $\left|\frac{d}{d t}\left\langle\eta, x_{n}(t) \xi\right\rangle\right|$ is bounded by $\left|K_{\eta \xi}\right|$ and each $x_{n}$ takes values in the compact set $x_{n}+T K_{\eta \xi}$. There exists a subsequence $x_{n_{k}}$ such that $x_{n_{k}}$ converges uniformly to $x($.$) on I$ and $\frac{d}{d t}\left\langle\eta, x_{n_{k}}(.) \xi\right\rangle$ converges to $\frac{d}{d t}\langle\eta, x(.) \xi\rangle$.

Fix $t_{1}$ and $t_{2}$ in $I$, and set $f_{k}(s)(\eta, \xi)=f_{n_{k}}\left(s, x_{n_{k}}(s)\right)(\eta, \xi), \Phi(s)(\eta, \xi)=$ $\mathbb{P}(s, x(s))(\eta, \xi)$.

$$
\begin{aligned}
\mathbf{d}\left(\left\langle\eta,\left(x\left(t_{2}\right)-\right.\right.\right. & \left.\left.\left.x\left(t_{1}\right)\right) \xi\right\rangle, \int_{t_{1}}^{t_{2}} \Phi(s)(\eta, \xi) d s\right) \\
\leq \mathbf{d}\left(\left\langle\eta,\left(x\left(t_{2}\right)-x\left(t_{1}\right)\right) \xi\right\rangle,\left\langle\eta,\left(x_{n_{k}}\left(t_{2}\right)-x_{n_{k}}\left(t_{1}\right)\right) \xi\right\rangle\right. & \\
& +\mathbf{d}\left(\left\langle\eta,\left(x_{n_{k}}\left(t_{2}\right)-x_{n_{k}}\left(t_{1}\right)\right) \xi\right\rangle, \int_{t_{1}}^{t_{2}} \Phi(s)(\eta, \xi) d s\right) .
\end{aligned}
$$

But

$$
\begin{gathered}
\mathbf{d}\left(\left\langle\eta,\left(x_{n_{k}}\left(t_{2}\right)-x_{n_{k}}\left(t_{1}\right)\right) \xi\right\rangle, \int_{t_{1}}^{t_{2}} \Phi(s)(\eta, \xi) d s\right) \\
=\mathbf{d}\left(\int_{t_{1}}^{t_{2}} f_{k}(s)(\eta, \xi) d s, \int_{t_{1}}^{t_{2}} \Phi(s)(\eta, \xi) d s\right) \\
=\inf \left\{\left|\int_{t_{1}}^{t_{2}} f_{k}(s)(\eta, \xi) d s-\int_{t_{1}}^{t_{2}} \varphi(s)(\eta, \xi) d s\right|: \varphi(.)(\eta, \xi) \in \Phi(.)(\eta, \xi)\right\} \\
\leq \inf \left\{\int_{t_{1}}^{t_{2}}\left|f_{k}(s)(\eta, \xi)-\varphi(s)(\eta, \xi)\right| d s: \varphi(.)(\eta, \xi) \in \Phi(.)(\eta, \xi)\right\} .
\end{gathered}
$$


Since $x_{n_{k}}(s) \rightarrow x(s)$ and $\Phi$ is upper semicontinuous,

$$
d_{\eta \xi}\left(\left((s, x), f_{n_{k}}(s)(\eta, \xi)\right), \operatorname{graph} \Phi\right) \rightarrow 0 ;
$$

it follows that $\mathbf{d}\left(f_{k}(s)(\eta, \xi), \Phi(s)(\eta, \xi)\right)$ converges pointwise to zero.

Then for each $k$ there exists $\varphi_{k}$ a measurable selection from $\Phi$, such that $\left|f_{k}(s)(\eta, \xi)-\varphi_{k}(s)(\eta, \xi)\right|=\delta\left(f_{k}(s)(\eta, \xi), \Phi(s)(\eta, \xi)\right)$ a.e.

Hence

$$
\begin{aligned}
\inf \left\{\int \mid f_{k}(.)(\eta, \xi)-\right. & \varphi(.)(\eta, \xi) \mid: \varphi(.)(\eta, \xi) \in \\
\left.\leq \int(.)(\eta, \xi)\right\} & \\
& =\int \delta\left(f_{k}(.)(\eta, \xi)-\varphi(.)(\eta, \xi) \mid\right.
\end{aligned}
$$

By Dominated convergence theorem, the integral converges to zero.

We have

$$
\mathbf{d}\left(\left\langle\eta,\left(x\left(t_{2}\right)-x\left(t_{1}\right)\right) \xi\right\rangle, \int_{t_{1}}^{t_{2}} \Phi(s)(\eta, \xi) d s\right)=0 .
$$

Since $T$ is arbitrarily chosen, from Lemma 13 , we conclude that $x($.$) is an$ adapted weakly absolutely continuous solution of (3.1).

\section{References}

[1] J.P. Aubin and A. Cellina, Differential Inclusions, Springer- Verlag, $\operatorname{Berlin}(1984)$.

[2] J.P. Aubin and H. Frankowska, Set valued Analysis, Springer- Verlag, Berlin(1990).

[3] E.O. Ayoola, Exponential formula for the reachable sets of quantum stochastic differential inclusions. Stoch. Anal. Appl.21(2003)3, 515-543. http://dx.doi.org/10.1081/SAP-120020424.

[4] E.O. Ayoola, Continuous selections of solution sets of Lipschitzian quantum stochastic differential inclusions.Int. J. Theor.Phys.43(2004)10, 20412059. http://dx.doi.org/10.1023/B:IJTP.0000049009.71965.co

[5] E.O. Ayoola, Topological properties of solution sets of Lipschitzian quantum stochastic differential inclusions.Acta Appl.Math100(2008), 15-37. http://dx.doi.org/10.1007/SI0440-007-9175-1 
[6] K. Deimling, Multivalued differential equations, Walter de Gruyter (1992).

[7] G.O.S. Ekhaguere, Lipschitzian quantum stochastic differential inclusions Int. J. Theor. Phys.31(1992)11, 2003-2034. http://dx.doi.org/10.1007/BF00671969.

[8] G.O.S. Ekhaguere, Quantum stochastic differential inclusions of hypermaximal monotone type Int. J. Theor. Phys.34(1995)3, 323-353. http://dx.doi.org/10.1007/BF00671595.

[9] G.O.S. Ekhaguere, Quantum stochastic evolutions Int. J. Theor. Phys.35(1996)9, 1909-1946. http://dx.doi.org/10.1007/BF02302422.

[10] G.O.S. Ekhaguere, Topological solutions of Non commutative stochastic differential equations Stoch. Anal. and Appl. 25(2007)9, 961-993. http://dx.doi.org/10.1080/07362990071540345.

[11] A. Gavioli, A viability result in the upper semicontinuous case J. convex Anal.5, (1998)(2), 381-395.

[12] A. Guichardet, Symmetric Hilbert spaces and related topicsLecture Notes in Mathematics, 261, Springer-Verlag, Berlin(1972).

[13] R.L. Hudson and K.R. Parthasarathy, Quantum Ito's formula and stochastic evolutionsComm. Math. Phys.93,(1984)3, 301-323. http://dx.doi.org/10.1007/BF01258530.

[14] M.O. Ogundiran and E.O. Ayoola, Mayer problem for Quantum stochastic control J. Math. Phys.51(2010) 1, 023521-023521-8. http://dx.doi.org/10.1063/1.3300332.

[15] K.R. Parthasarathy, An introduction to Quantum stochastic calculus, Monographs in Mathematics 85, Birkhauser Verlag, Basel(1992). 
